\title{
Zur Bestimmung der Halogene organischer Körper.
}

(Mit 9 Holzschnitien.)

\section{Von Carl Zulkowsky und Carl Lepéz.}

Seitdem Kopfer in dem Platin einen bequemen Sauerstoffiibertrager für die Elementaranalyse gefunden, ${ }^{1}$ haben Andere diese werthvolle Eigenschaft des Platins noch weiter auszunützen gesucht. So unter Anderen Weidel und Schmidt für die Bestimmung des Schwefels organischer Körper ${ }^{2}$ und einer von uns zur Ermittlung des nutzbaren Schwefels der Lamming'schen Masse und der Kiese. ${ }^{3}$ Auf dieselbe Eigenschaft grïndet auch Winkler sein Verfahren der Darstellung von Schwefelsäureanhydrid.

Wir stellten uns nunmehr die Frage, ob die Kopfer'sche Art der Verbrennung organischer Körper nicht etwa zur Bestimmung der Halogene verwerthet werden könnte. Diese Idee ist iibrigens nicht neu und es hat bereits $\mathrm{Kopfer}$ diesbezügliche Versuche angestellt, welche aber von keinem günstigen Erfolge begleitet waren. ${ }^{4}$ Trotz dieser wenig einladenden Ergebnisse hofften wir die Kopfer'sche Methode auch diesem Zwecke anpassen zu können.

\section{Einleitende Versuche.}

Kopfer benützte für seine Art der Elementaranalyse ein Gemisch von Asbest und Platinschwarz, Winkler empfahl zur Oxydation des Schwefeldioxyds platinirten Asbest und letzterer

1 Zeitschrift für analyt. Chemie. 17. Jahrgang. S. 1.

2 Berliner Berichte. 1877. S. 1131.

3 Berichte der öst. Gesellschaft zur Förderung der chem. Industrie. 1881. S. 2.

4 Zeitschrift für analyt. Chemie. 17. Jahrgang. S. 23. 
bewährt sich in der That vortrefflich bei der Bestimmung des Schwefels; trotzdem ist die Anwendung von Asbest im vorliegenden Falle nicht unbedenklich. Es ist dje Möglichkeit vorhanden, dass die Halogene von den basischen Bestandtheilen zum Theil zurïckgehalten werden. Platinirter Asbest hat ausserdem die unangenehme Eigenschaft, bei längerem Gebrauche in ein Pulver zu zerfallen, dessen Anwendung so manche Schwierigkeit verursacht. Noch schlechter erwies sich mit Salzsäure ausgekochte und platinirte Chamotte, indem dieselbe die Halogene ziemlich stark zurückhält, so dass wir schliesslich zum Quarze greifen mussten. Gekörnter und platinirter Quarz bietet allerdings den Gasen keine so grosse Oberfläche dar als der faserige Asbest, allein er ist trotzdem wirksam genug, um die Verbrennung, bei Einhaltung gewisser Vorsichtsmassregeln, anstandslos durchzuführen.

\section{Darstellung von platinirtem Quarz.}

Reinster Quarzit wird ausgeglüht und im glühenden Zustande mit Wasser abgeschreckt, um ihn mürber und poröser zu gestalten. Die Stücke zerschlägt man in einem eisernen Mörser bis zur Grösse eines Hanfkornes, siebt das feine Pulver als auch die grösseren Stückchen ab und zieht das Brauchbare mit Salzsäure aus, um das anhaftende Eisen zu entfernen.

Zum Platiniren nimmt man auf 100 Theile gekörnten Quarz $4-5$ Theile reinstes aus Platinsalmiak dargestelltes Platin, ${ }^{1}$ löst dasselbe in Königswasser und dampft den Überschuss des letzteren auf dem Wasserbade ab. Der Riickstand wird in so viel Alkohol gelöst, dass diese Lösung eben hinreicht, den gekörnten Quarz zu bedecken. Nachdem der letztere mit der Platinlösung in einer Schale tibergossen, wird der Alkohol auf dem Wasserbade verdampft und der Rückstand auf dem Sandbade zur völligen Trockniss gebracht. Hierauf wird derselbe in ein Rohr gefiullt und im Wasserstoffstrome ansgeglïht. Mit käuflichem Platinchlorid oder käuflichem Platin haben wir die schlimmsten Erfahrungen gemacht, weil dieselben stets Kupfer und Eisen enthalten und die Halogene zurickhalten.

${ }_{1}$ Der Platinsalmiak wird im Schiffehen in einem Glasrohr für sich erhitzt and erst darnach Wasserstoff darübergeleitet. 
Wahrnehmungen bei der Verbrennung halogenhaltiger Koblenstoffverbindungen vermittelst platinirten Quarzes.

Wenn man eine halogenhaltige organischeSubstanz in später zu beschreibender Weise im Sauerstoff und unter Zuhilfenahme von gekörntem platinirtem Quarze verbrennt, so lassen sich hierbei verschiedene Wahrnehmungen machen.

a) Ist die Substanz jodhaltig, so entweicht das Jod mit den sonstigen Verbrennungsproducten (Kohlensäure, Wasser) im freien Zustande. Die Abspaltung des Halogens kann möglicherweise mehrere Zwischenstadien durchlaufen, das Endresultat ist aber inmer freies Jod, so dass man an der Farbe der auftretenden Dämpfe das Jod erkennt. Es gibt wohl keine einfachere und sicherere Methode, das Jod einer organischen Verbindung qualitativ nachzuweisen, als eben diese.

b) Ist die Substanz brombaltig, so entweicht das Brom mit den sonstigen Verbrennungsproducten vorwiegend im freien Zustande $(95-99 \%)$ und der kleine Rest als Bromwasserstoff.

c) Ist die Substanz chlorhaltig, so entweicht das Chlor theils als solches, theils als Chlorwasserstoffsäure. Obwohl das Verhältniss beider nicht ermittelt wurde, so ist es aus theoretischen und sonstigen Gründen sehr wahrscheinlich, dass die Wasserstoffverbindung dieses Halogens sehr schwer verbrennt und daher in grösserer Menge auftreten dürfte. Wenn das Halogen nur im freien Zustande oder nur als Wasserstoffverbindung auftritt, so ist dessen Bestimmung sehr leicht durchfiihrbar. Man braucht eben nur ein solches Absorptionsmittel vorzuschlagen, welches für die eine oder die andere Form des Halogens geeignet ist. Weit schwieriger gestaltet sich die Sache, wenn das Halogen im freien Zustande und als Wasserstoffverbindung auftritt. In diesem Falle ist man in der Wahl der Absorptionsmittel ausserordentlich beschränkt und es ist fast zu verwundern, dass uns die Auffindung einer hiefür brauchbaren Substanz gelang. Die Schwierigkeit lässt sich begreifen, wenn man bedenkt, dass dieses Absorptionsmittel sowohl das freie als 
auch das gebundene Halogen in eine einheitliche Substanz überfuhren muss, welche eine gen au e quantitative Bestimmung desselben leicht zulässt.

\section{Über die Absorptionsmittel zur Bestimmung der} Halogene.

Ist die Substanz jodhaltig, so kann zum Auffangen des freien Jods eine Lösung von Jodkalium benützt werden. In diesem Falle gestaltet sich die Bestimmung des Jods ungemein einfach, indem der Gehalt dieser Lösung auf massanalytischem Wege mit thioschwefelsaurem Natron ermittelt werden kann.

Ein zweites, anch für die anderen Halogene verwendbares Absorptionsmittel ist eine mit Ammoniak versetzte Lösung von Wasserstoffhyperoxyd. Letzteres soll möglichst rein, vornehmlich chlorfrei sein und liefert dasselbe die Firma H. Trommsdorff in Erfurt als $3 \%$ Lösung, welche im kühlen und dunklen Raume aufbewahrt werden muss, um eine Zersetzung möglichst hintanzuhalten. ${ }^{1}$ Eine Untersuchung dieses Präparates ergab einen sehr kleinen, aber doch bestimmbaren Chlorgehalt, so dass man für genaue Bestimmungen denselben in Rechnung bringen muss.

Es ergaben 25 Cc. $0 \cdot 0032 \mathrm{Grm}$. Chlorsilber, und wenn man für die Absorption dieses Volum benöthigt, so wird das Gewicht des Chlorsilbers von dem Gewichte der erhaltenen HalogenSilberverbindung in Abzug gebracht.

Wenn das Wasserstoffhyperoxyd durch längeres Lagern seinen Gehalt merklich eingebüsst hat, was man durch eine einfache Titration nach A sch of $f^{2}$ leicht ermitteln kann, so muss für die Absorption eine grössere Menge verwendet werden. Die Reaction, welche das Jod auf das Absorptionsmittel ausuibt, vollzieht sich in zwei Phasen, zum Beispiel:

$$
\begin{aligned}
\mathrm{J}_{2}+\mathrm{H}_{2} \mathrm{O}_{2} & =2 \mathrm{HJ}+\mathrm{O}_{2} \\
2 \mathrm{JH}+2 \mathrm{NH}_{3} & =2 \mathrm{NH}_{4} \mathrm{~J} .
\end{aligned}
$$

Das Jod verschwindet augenblicklich unter Auftreten von Sauerstoffbläschen. Man hat nur Sorge zu tragen, dass eine

1 Am 1. Mai enthielt das frische Präparat $3.28 \%$ und am 8. Juli waren noch $1.94 \%$ Wasserstoffsuperoxyd vorbanden.

$\approx$ Fresenius quant. Analyse. 6. Auflage. S. 584. 
genügende, etwa die doppelte oder dreifache Menge von Hyperoxyd in der vorgeschlagenen Flüssigkeit enthalten sei.

Ist die zn untersuchende Substanz brom- oder chlorhaltig, so kann man als Absorptionsmittel nur die mit Ammoniak versetzte Lösung des Wasserstoffhyperoxyds gebrauchen.

Das Brom und Chlor treten wie bekannt im freien Zustande und als Hydrüre auf. Erstere reagiren auf das Hyperoxyd wie das Jod, letztere verbinden sich einfach mit dem Ammoniak. Das Endergebniss besteht in der Bildung von Brom- und Chlorammonium.

Aus allen diesen Flüssigkeiten lässt sich das Halogen auf bekannte Weise mit salpetersaurem Silber herausfällen und dessen Menge bestimmen.

Zur quantitativen Bestimmung sämmtlicher Halogene ist wohl noch ein zweites Absorptionsmittel denkbar, welches wir aber bis jetzt noch nicht versucht haben, weil dasselbe keine so grosse Genauigkeit zulassen dürfte. Dasselbe wäre eine titrirte Lösung von Ätzkali oder Ätznatron. Wendet man eine gemessene und iberschilssige Menge solcher Lösungen an, so wirkt das Chlor je nach der Concentration und Temperatur auf zweifache Weise ein:

I.

$$
2 \mathrm{KOH}+2 \mathrm{Cl}=\mathrm{KCl}+\mathrm{KOCl}+\mathrm{H}_{2} \mathrm{O}
$$

II.

$$
6 \mathrm{KOH}+6 \mathrm{Cl}=5 \mathrm{KCl}+\mathrm{KClO}_{3}+3 \mathrm{H}_{2} \mathrm{O}
$$

Aus diesen Gleichungen ergibt sich, dass ein Atom Chlor in beiden Fällen ein Molekül Ätzkali beansprucht. Wenn man nun den iiberschïssigen Rest des Ätzkalis alk alimetris ch bestimmt, so lässt sich durch Rechnung sehr leicht die Menge des Chlors bestimmen. Nachdem das Brom und Jod gegen Alkali dasselbe Verhalten zeigen, so liessen sich auch diese Halogene auf gleiche Weise bestimmen. Aber auch für den qualitativen Nachweis des Broms und des Chlors ist die Verbrennungsmethode sehr zu empfehlen, da sich dieselbe für diesen Fall sehr vereinfachen lässt. Man braucht hiefür kein Wasserstoffhyperoxyd, sondern nur eine chlorfreie Lösung von Soda, Pottasche oder selbst nur Wasser vorzuschlagen, um die Natur des Halogens festzustellen. 
Beschreibung des Verbrennungs- und Absorptionsapparates.

Nachdem sich die halogenhaltigen Kohlenstoffverbindungen bei der Verbrennung ungleich verhalten, das Jod im freien Zustande, das Brom und Chlor hingegen theils als solche, theils als Hydrüre auftreten, so verwendet man nicht in allen Fällen

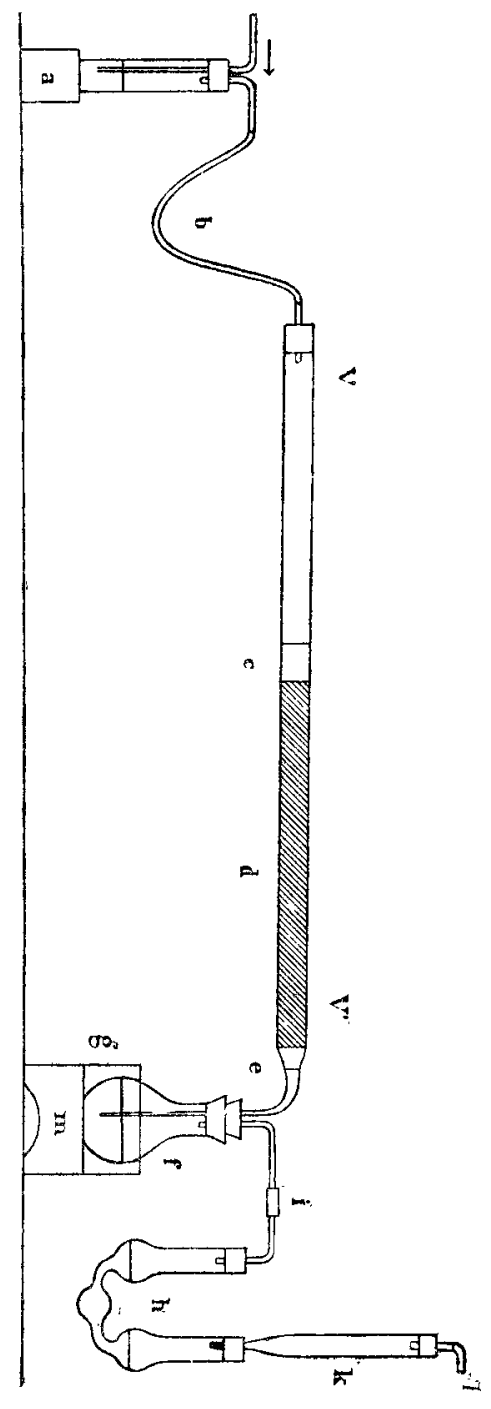

denselben Verbrennungs- und Absorptionsapparat, obzwar sich der Unterschied bei ersterem nur auf die Dimensionen beschränkt. Ebenso sind verschiedenartige Hilfsvorrichtungen nöthig, je nachdem die Substanz fest oder flüssig, leicht oder schwer flüchtig ist.

Die Einrichtung des gewöhnlichen Apparates ergibt sich aus Fig. 1.

$a$ Indicator mit Wasser gefiullt, welcher mit dem Sauerstoffgasometer in Verbindung steht und die Stärke des Gasstromes anzuzeigen hat.

$b$ Verbindungsschlanch.

$V V^{\prime}$ Verbrennungsrohr aus schwer schmelzbarem Glase, welches zu einer langen,nach abwärts gebogenen Spitze ausgezogen erscheint. Darin ist:

$c$ Eine Platinblechrolle, um dem gekörnten Platinquarz einen Halt zu geben.

d Platinirter Quarz.

$e$ Ein lockerer Pfropf von langfaserigem Asbest, nm das Verstäuben von Platin zu verhüten.

$f$ Ein Kölbchen, etwa 80-100 Cc. fassend.

$g$ Ein kleines Glasschälchen, welches mit kalten Wasser gefüllt wird, nm einer Erhitzung und der hiedureh bewirkten Zersetzung des Wasserstoffsuperoxydes vorzubeugen. 
$h$ Peligotröhre mit dem Kölbchen durch ein kurzes Kautschuckröhrchen $i$ verbunden.

$l$ Rohraufsatz, mit Glaswolle gefüllt und bei $l$ mit einem kleinen Glasröhrchen versehen.

$m$ Ein Steg aus Pappendeckel oder dünnen Brettchen zur Unterstiutzung. $f$, $h$ und $k$ dienen zur Absorption.

Zum Erhitzen des Verbrennungsrohres wird ein Erlenmeyer'scher (nicht Glaser'scher) Ofen verwendet. Nachdem dasselbe kürzer als der Ofen ist, so muss diejenige Thonrinne, auf welcher das Rohrende $V$ aufruht, hinweggenommen werden. Ein kürzerer Ofen wäre jedenfalls zweckmässiger, da dieses Rohrende innerhalb des Ofens schwerer zugänglich ist und deshalb Unbequemlichkeiten in der Handhabung verursacht.

Das Verbrennungsrohr wird in den Ofen so eingesetzt, dass das rechtsseitige Ende mit der abgebogenen Spitze möglichst wenig herausragt, damit der Asbestpfropf ins Glühen kommen kann. Leider reicht bei dem Erlenmeyer'schen Ofen die Brennerreihe nicht so weit, so dass man genöthigt ist, einen separaten Bunsen'schen Brenner seitlich einzuschieben, der sich zwischen den Verbindungsstangen festklemmt und in dieser Lage ohne jedwede Stuitze erhält, wie dies aus folgender rechtsseitiger Ansicht hervorgeht. (Fig. 2.) Das Gas für denselben kann dem Gasrohre des Ofens am rechten Ende entnommen werden.

Fig. 2.

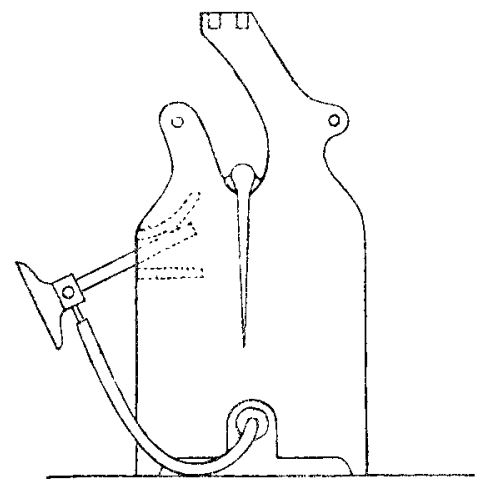

Verfahren bei festen oder flüssigen Körpern, welche schwer flüchtig sind.

Man beginnt die Analyse mit der Füllung des Absorptionsapparates. $\mathrm{Zu}$ diesem $\mathrm{Zwecke}$ bereitet man sich die zu dem Ver- 
suche nöthige Menge der Absorptionsflitssigkeit, indem man $25-30$ Ce. Wasserstoffsuperoxyd (von cirea $3 \%$ Gehalt) mit 5 Ce. concentrirtem Ammoniak vermischt und mit diesem Gemenge sowohl das Kölbchen $f$ und die Peligotröhre $\boldsymbol{h}$ beschickt, als auch die im Rohraufsatz $k$ befindliche Glaswolle tränkt. Enthält das Wasserstoffsuperoxyd bestimmbare Mengen von Chlor, so muss natürlich mit gemessenen Mengen desselben gearbeitet und das Resultat in früher beschriebener Art corrigirt werden. Hierauf wird die Spitze des Verbrennungsrohres mit dem Kölbchen so vereinigt, dass dieselbe in die Fliissigkeit eintaucht. Das Kölbchen wird in die kleine, mit kaltem Wasser gefüllte Glasschale eingesenkt und das Ganze anf den Steg gesetzt. Hat man das Verbrennungsrohr in die richtige Lage gebracht, das heisst so tief als möglich in den Ofen eingesetzt, so wird das Stiick $c e$ angeheizt und zum hellen Rothglühen gebracht. Während des Anheizens lässt man entweder das Rohrende $V$ offen und dann kann man die Peligotröhre gleich ron Anfang an mit dem Kölbchen $f$ fest verbinden, oder man schliesst $V$ und leitet einen langsamen Sauerstoffstrom hindurch; dann muss jedoch die Peligotröhre erst mitverbunden werden, wenn die Substanz bereits in das Rohr eingeführt ist. Es setzt nämlich die Sperrflüssigkeit in $h$ und $k$ dem Gasstrome einen Widerstand entgegen und dieser bewirkt ein Anwachsen des Druckes in f: Da nun beim Einfiihren der Substanz der Stöpsel bei $V$ entfernt werden muss, so würde in diesem Momente durch den Überdruck die Sperrfluissigkeit in das Rohr empor gehoben werden und ein Zerspringen desselben herbeiführen.

Die zn analysirende Substanz wird in einer Menge von $1 / 4-1 / 2$ Grm. abgewogen. Ist dieselbe fest und schwer flüchtig, so wird sie in ein Porzcllanschiffchen eingewogen. Ist sie fliissig und schwer flüchtig, so wiegt man selbe in einem Glasröhrchen ab,

Fig. 3.

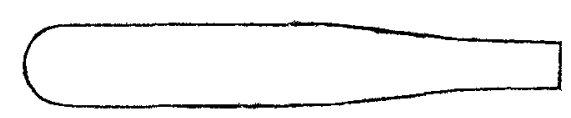

welches beistehend (Fig. 3) in Naturgrösse abgebildet ist und welches man bei der Wägung und dem Transporte mit einem 
Kautschuckröhrchen mit eingestecktem Glasstabe verschlossen hält. Auch lässt sich statt dessen ein Röhrchen mit eingeschliffenem Stöpsel verwenden. Selbstverständlich dürfen diese Verschlussvorrichtungen nicht mit der Flüssigkeit in Beruhrung kommen, weil sie nicht in das Verbrennungsrohr eingefuhrt werden; ferner muss die Füllung mit Hilfe einer feinen Saugpipette erfolgen. Aus Kügelchen mit capillarer Spitze, wie sie sonst bei der Elementaranalyse von Flüssigkeiten üblich sind, kann kein gleichförmiges und ruhiges Verdampfen erzielt werden; daher sind dieselben hier nicht anwendbar.

Sollte die Substanz fest, aber doch so weit flüchtig sein, dass nach der Abwage bis zur Einführung in das Verbrennungsrohr ein wägbarer Verlust zu erwarten stuinde, so benützt man zum Wägen ebenfalls obiges Röhrchen.

Wurde die Substanz in ein Röhrchen eingewogen, so wird dasselbe unmittelbar vor der Einführung in das Verbrennungsrohr geöffnet und anf ein Porzellanschiffehen etwas schräg gestellt. Die Beschickung des Verbrennungssohres erfolgt immer dann, sobald die Schichte des platinirten Quarzes gehörig glüht. Das bereit gehaltene Schiffchen wird darnach so weit eingeschoben, dass dasselbe 8-10 Ctm. von der Platinblechrolle entfernt ist, das Rohrende $V$ sofort geschlossen und falls dies nicht schon früher geschehen, das Peligotrohr sammt Aufsatz mit dem Kölbchen $f$ verbunden. Sodann leitet man dem Verbrennungsrohre per Minute circa 25 Cc. Sauerstoff zu und behält diese Geschwindigkeit während der ganzen Verbrennung bei. Sobald bei $l$ Sauerstoff austritt, kann zar eigentlichen Verbrennung geschritten werden. Man zündet je nach der Flüchtigkeit der zu analysirenden Substanz in grösserer oder geringerer Entfernung links vom Schiffehen ein kleines Flämmchen an und bedeckt das Rohr an dieser Stelle mit einem Ziegel. Der übrige Theil des Rohres, soweit dasselbe nicht erhitzt wird, bleibt unbedeckt, um dasselbe möglichst kalt zu erhalten. Die Temperatur wird nun durch allmählige Vergrösserung der Flamme so regulirt, dass eine langsame und gleichförmige Verdampfung oder Zersetzung der eingeführten Substanz stattfindet.

In keinem Falle darf die Flüssigkeit oder die geschmolzene Substanz ins Sieden kommen, da sonst der Versuch unbe- 
dingt verloren ist, eventuell auch eine Explosion stattfinden kann. ${ }^{1}$

Bei der Regulirung der Temperatur leistet auch das Vertauschen der heisseren Ziegeln, welche den glühenden Theil des Rohres bedecken, gegen die kälteren, die sich tiber der Substanz befinden, oft gute Dienste. Specielle Regeln lassen sich hier selbstverständlich nicht aufstellen, da Körper von verschiedenem Siedepunkte und verschiedener Zersetzbarkeit ein abweichendes Arbeiten verlangen.

Man hat sich stets gegenwärtig zu halten, dass die Verbrennung nur mit dem zugeleiteten Sauerstoff bewirkt wird, dass somit dieser in jedem Momente überschüssig sein muss.

Tritt einmal der Fall ein, dass mehr fluchtige Producte entwickelt werden, als der zuströmende Sauerstoff zu verbrennen vermag, so ist das Überdestilliren unverbrannter Substanz unausweichlich. Den Eintritt der Destillation erkennt man sofort an dem rechten Rohrende, welches sich mit den Destillationsproducten beschlägt; auch nehmen die Austrittsgase einen eigenthtimlichen brenzlichen Geruch an, der trotz der Gegenwart des Ammoniaks erkennbar ist.

Der Beginn der Verbrennung ist bei jodhaltigen Substanzen durch die violette Färbung der Austrittsproducte, bei brom- und chlorhaltigen Körpern durch das Auftreten von Nebeln oberhalb der Absorptionsflïssigkeit charakterisirt. Bei Jodverbindungen macht sich die Nebelbildung nur sehr schwach bemerkbar. Zum Zurückhalten dieser Nebel dient die Glaswolle, ohne welche diese Verbrennungsmethode tiberhaupt gar nicht durchführbar wäre. Die Anstrittsgase, welche dieselbe passiren, dürfen natürlich nicht die geringste Spur eines Nebels zeigen.

Obwohl diese Nebel die Verdichtung erschweren, so sind sie anderseits eine willkommene Erscheinung, weil sie den Beginn und das Ende der Verbrennung anzeigen. Deshalb wählten wir Ammoniak und kein Ätzkali, auch wenn selbes leicht chlorfrei zu beschaffen wäre.

1 Dieser Gefahr kann übrigens dadurch vorgebeugt werden, dass man den sonst leeren Raum zwischen Schiffchen und Platinrolle mit gekörntem Quarz anfüllt. 
Die Verkohlung, Verdampfung oder Verbrennung der Substanz kann in den meisten Fällen mit einer einzigen Flamme zu Ende geführt werden; selten wird noch eine zweite, dem Schiffchen nähere Flamme nothwendig sein.

Ist alle Substanz verbrannt, so bringt man auch den, bisher nicht erhitgten Theil des Rohres zum Glühen und wartet so lange, bis die in das Kölbchen $f$ eintretenden Gasblasen keine Nebelbildung mehr bewirken. Dieser Punkt ist, von dem Momente an gerechnet, wo das ganze Rohr glüht, bei Jodverbindungen in circa 10 Minuten, bei Bromverbindungen in circa 15 Minuten erreicht. Bei chlorhaltigen Substanzen macht sich ein grosser Unterschied bemerkbar, weil die, wenn auch schwache Nebelbildung noch nach $1 / 2-1$ Stunde wahrnehmbar ist. Das Platin scheint nach Allem das Chlor oder die Chlorwasserstoffsäure mit grosser Kraft zurïckzuhalten; man muss sich also begnügen, als Ende der Operation jenen Zeitpunkt anzusehen, bei welchem die Nebelbildung auf ein gewisses Minimum gesunken ist.

Verfahren bei leichtflüchtigen Flüssigkeiten.

Dieselben lassen sich nicht direct in das Rohr einführen, weil trotz aller Gegenmassregeln eine stärkere Verdampfung stattfindet, als der Sauerstoffzufuhr entspricht. Nach mehrfachen missgltickten Versuchen wählten die Verfasser das nachfolgend beschriebene Verfahren, welches auch in diesem Falle ein vollkommen sicheres Arbeiten gestattet.

Das Princip desselben besteht darin, dass ausserhalb des Verbrennungsrohres über die zu analysirende Flüssigkeit ein regulirbarer, langsamer Sauerstoffstrom geleitet wird. Der mit Flüssigkeitsdampf beladene Sauerstoff tritt mit einem stärkeren und überschïssigen Sauerstoffstrom glejchzeitig in das Rohr zur Verbrennung ein.

Hiezu sind folgende Geräthe erforderlich:

A. Vorrichtung zur Zweitheilung des Sauerstoffstromes (Fig. 4).

Der Sauerstoff des Gasometers tritt vermittelst eines Schlauches bei $f$ in das Gabelrohr ein, theilt sich in demselben in zwei Ströme, von welchen einer durch $c$, der zweite durch $c_{1}$ geftuhrt wird. Der Austritt erfolgt bei $e$ und $e_{1}$, und zwar in ver- 
schiedener Stärke, je nachdem man den einen oder den andern Zweigstrom stärker drosselt. Dadurch lässt sich die Verdampfungsgeschwindigkeit der zu analysirenden Flïssigkeit vollkommen beherrschen.

Fig. 4.

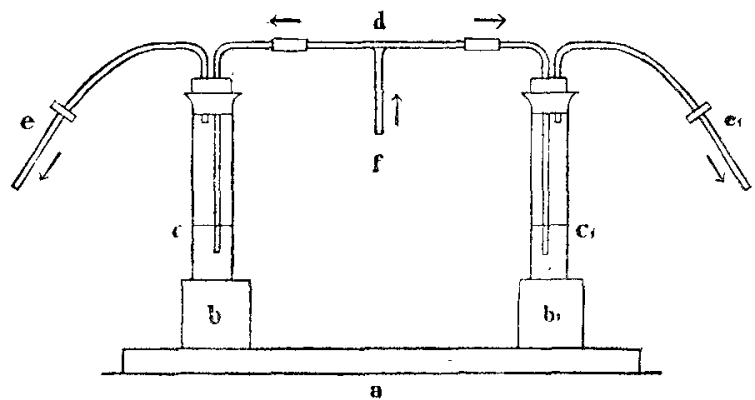

a Holzbrettchen.

$b b^{\prime}$ Ausgehöhlte Korkstöpsel, welche als Träger dienen.

c Eprouvette mit Wasser gefüllt und als Indicator dienend.

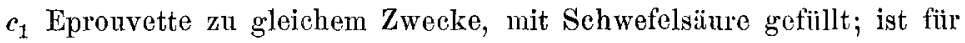
denjenigen Saterstoffstrom bestimmt, welcher ïber die Sulstanz geleitet wird. Die Schwefelsäure trocknet denselben und verhiutet einen Beschlag von Feuchtigkeit in dem Flïssigkeitsbehälter.

$d$ Gabelrohr für die $Z_{\text {weitheilung des Sauerstoffstromes. }}$

$e e^{\prime}$ Kautschuckröhrchen mit Schraubenquetschern, welche mit dem Stöpsel des Verbrennungsrohres communieiren.

B. Kugelröhrchen zum Abwägen der Substanz in beistehend gezeichneter Form und halber Grösse (Fig. 5). Dasselbe wird aus einem Glasröhrchen von passender Stärke durch Aufblasen des mittleren Theiles $n$ und nachheriges Biegen und Auszielien hergestellt.

Fig. 5.

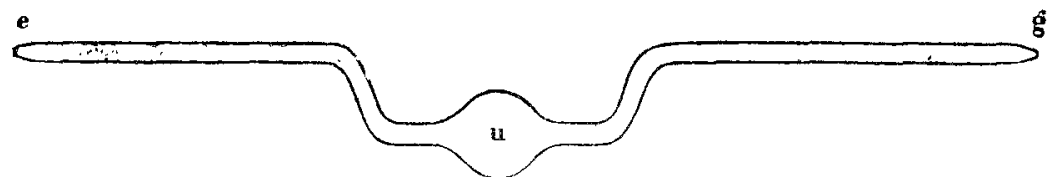

Man bestimmt die Tara des Kugelröhrchens, saugt mit Hilfe eines Schlauches eine passende Menge der fraglichen Flüssigkeit ein und trocknet den in dieselbe eingetauchten Theil durch gelindes Erwärmen über einer heissen Platte. Die beiden Endspitzen werden zugeschmolzen, das gefüllte Kugelrohr gewogen und hieraus ergibt sich das Gewicht der eingeführten Flüssigkeit. 
Die Zweitheilung des Sauerstoffstromes erfordert, dass der Stöpsel des Verbrennungsrohres mit zwei Bohrungen versehen ist. In die eine kommt das Kugelrohr, dessen Spitzen vorher mit einem Diamant geritzt wurden, in die zweite ein dickwandiges Röhrchen, dessen Ende $h$ (Fig. 6) so weit abgebogen wird, dass beim Drehen desselben in der Bohrung die Spitze $g$ erfasst werden kann, ohne die Wand des Verbrennungsrohres zu streifen. Zur

Fig. 6.

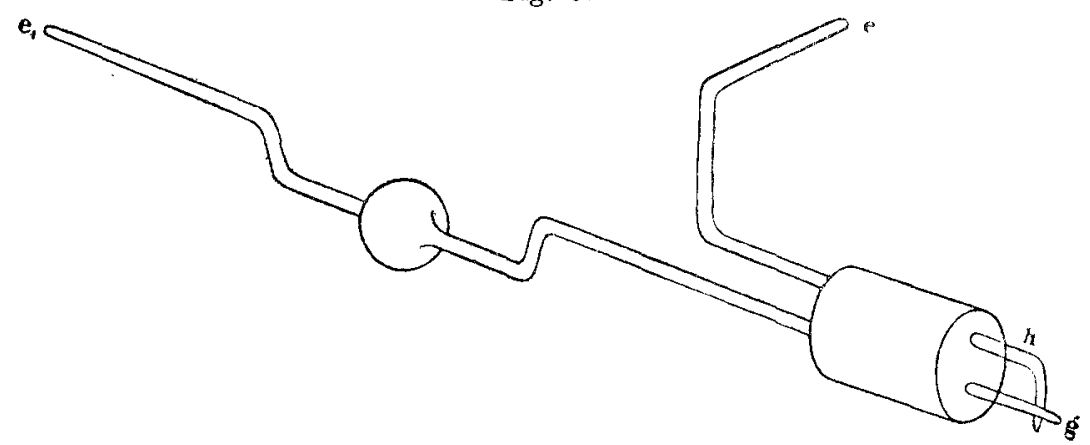

Vornahme der Verbrennung werden die Absorptionsapparate in schon beschriebener Weise gefüllt und vorgelegt. Das Verbrennungsrohr wird mit dem ausgerïsteten Stöpsel geschlossen und die beiden Röhrchen $e h$ und $e_{1} g$ mit dem Apparate zur Zweitheilung des Sauerstoffstromes verbunden. Man leitet anfänglich nur durch $e h$ einen langsamen Sauerstoffstrom zu. Die Quarzschichte wird hierauf angeheizt and sobald der Absorptionsapparat mit Sauerstoff gefullt ist, kann die Verbrennung beginnen. Man verstärkt zunächst den Sauerstoffstrom auf das iibliche Mass, dreht das Zuführungsrohr $e h$ so weit, dass die Spitze $g$ abbricht, worauf man dasselbe wieder in seine friihere Lage bringt. Ebenso wird die zweite Spitze des Kugelrohres $e_{1}$, welche im Kautschukschlauche steckt, abgebrochen. Der Quetschhahn dieses Schlauches wird nun vorsichtig so weit gelüftet, dass ein sehr langsamer Sauerstoffstrom die Kugelröhre passirt und in dem zugehörigen Indicator eine Blase in etwa 2-3 Secunden auftritt. Nunmehr ist die Verbrennung in Gang gesetzt, welche weiter keine besondere Aufmerksamkeit erfordert. Ist die Substanz in dem Kugelrohr bis auf einen kleinen Rest verdampft, so kann selbstverständlich der dasselbe passirende Sauerstoffstrom verstärkt 
werden. Ist jeder Rest von Flï.ssigkeit versehwunden, so lässt man die Hauptmenge des Sauerstoffes in die Kugelröhre eintreten, während man den andern Zweigstrom reducirt. Nunmehr wird das ganze Verbrennungsrohr angeheizt und die Verbrennung wie sonst zu Ende gefuhrt.

Nach diesem Verfahren wurden folgende Substanzen analysirt: Jodmethyl, Jodäthyl und Bromäthyl. (S. Beleganalysen.)

Specielles Verfahren bei der Analyse ron Jodverbindungen.

Die Jodverbindungen lassen sich sehr leicht verbrennen, bediirfen nicht viel von platinirtem Quarz, also nur ein kleineres Verbrennungsrohr. Die erforderlichen Dimensionen in Centimetern sind aus folgender Zeichnung zu entnehmen. (Fig. 7.)

Fig. 7.

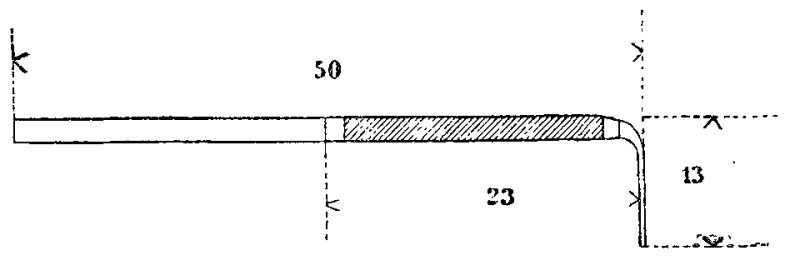

Nach Beendigung der Verbrennung verlangsamt man den Gasstrom und lässt das Rohr etwas erkalten, bevor man zur Entleerung der Vorlagen schreitet. Ein Theil des Jods setzt sich in der nach abwärts gebogenen Spitze in Form kleiner Krystalle an. Um diese herabzuspiilen und rasch in Lösung zu bringen, bläst man mit Hilfe eines bei $l$ (s. Fig. 1) angesetzten Kautschukröhrchens vorsichtig Luft ein, so dass die Supcroxydlösung in die Spitze emporsteigt. Sobald man zu blasen aufgehört, sinkt die Flüssigkeit rasch wieder zurück und reisst gleichzeitig die lockerer haftenden Jodtheilchen mit sich. Diese lösen sich nun leicht unter lebhafter Sauerstoff-Entwicklung auf. Das Ausspülen wird so lange wiederholt, bis alles Jod entfernt und in Lösung tiberfuiht ist. Sollte der Fall eintreten, dass nicht genug Superoxyd vorhanden wäre, um sämmtliches Jod zu lösen, so wird die Peligotröhre abgelöst, der Stopfen des Kolbens gelüftet, etwas Superoxyd zugesetzt und direct in denselben Luft bei $i$ mittelst 
eines Schlauches eingeblasen. Die Genauigkeit des Resultats wird bei ungenügender Menge von Superoxyd nicht beeinträchtigt, weil durch Einwirkung von Jod auf das jedenfalls iiberschüssige Ammoniak kein Jodat gebildet wird, sondern Jodstickstoff, eventuell Jodamin entsteht, welche bei weiterem Zusatze von Superoxyd Jodammonium liefern.

Ist das Ausspiten der Rohrspitze beendet, so wird der Absorptionsapparat von dem Verbrennungsrohre entfernt und erst jetzt der langsame Sauerstoffstrom unterbrochen, weil sonst leicht ein Zurücksteigen der Flüssigkeit eintreten könnte. Der Absorptionsapparat wird demontirt, die Flïssigkeit zusammengespült und die Spitze des Verbrennungsrohres aus- und inwendig gereinigt. Die vereinigten Absorptions- und Waschflüssigkeiten werden in einer Schale auf dem Wasserbade erhitzt, um das Ammoniak und Ammoncarbonat möglichst zu verjagen und das Ganze auf ein kleineres Volum zu bringen. Alsdann setzt man Silbernitrat und zuletzt Salpetersäure, zu um das Jod zu fällen. Die Beschreibung der weiteren Operationen ist für den Analytiker überflüssig.

Nachdem das Jod bei der Verbrennung quantitativ als solches erhalten wird, so lässt sich die Jodbestinmung dadurch wesentlich vereinfachen, dass man statt des Superoxydes und Ammoniaks Jodkalium vorschlägt und das aufgenommene Jod massanalytis ch bestimmt.

Der hiefür erforderliche Absorptionsapparat (Fig. 8) erhält folgende Einrichtung:

Fig. 8

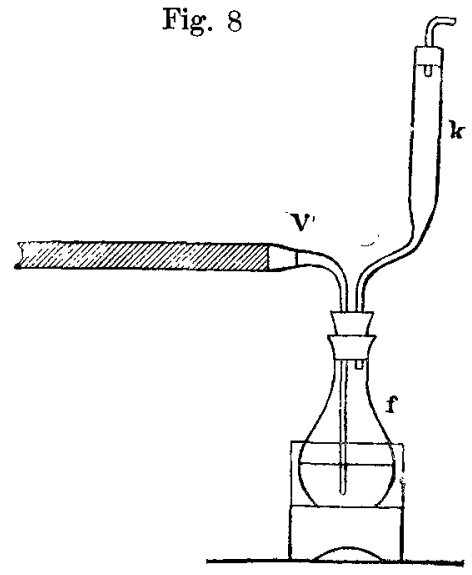

$V^{\prime}$ Ende des Verbrennungsrohres.

$f$ Kölbchen in früherer Grösse mit 20-25 Cc. einer 20procentigen Jodkaliumlösung gefüllt.

k Rohraufsatz mit Glaswolle beschickt, die mit derselben Jodkaliumlösung befeuchtet wird. 
Das rechtsseitige Abbiegen des Rohres $k$ hat den Zweck, dasselbe der Ofenbitze möglichst zu entziehen. Statt dessen könnte man anch zwischen Ofen und Rohr einen Schirm aus Pappe einschalten.

Ist die Verbrennung beendet, so wird zum Lösen der in der Rohrspitze befindlichen Jodkrystalle bei langsamem Sauerstoffstrome ebenfalls Luft eingeblasen. Die braune Jodlösung sammt Waschfliissigkeit wird dann mit $1 / 10$ thioschwefelsaurem Natron in bekannter Art titrirt. Im Nachstehenden sind die Ergebnisse der Beleganalysen verzeichnet, wobei bemerkt werden muss, dass es oft sehr schwierig war, geeignete Substanzen in absolut rein em Zustande zu erhalten. In solchen Fällen wurde der Jodgehalt nicht durch Rechnung, sondern durch die bisher anerkannt beste Methode von Carius ermittelt. Die aus der Theorie oder nach Carius gefundenen Zahlen stimmen mit den durch Verbrennung gefundenen auf das Beste tiberein, wie dies aus nachfolgender Tabelle hervorgeht. 
Zur Bestimmung der Halogene organischer Körper.

\begin{tabular}{|c|c|c|c|c|c|c|c|c|}
\hline \multirow{3}{*}{$\begin{array}{l}\text { Einwage } \\
\text { in } \\
\text { Grammen }\end{array}$} & \multicolumn{4}{|c|}{ Jod $g$ e halt } & \multicolumn{4}{|c|}{$\begin{array}{l}\text { Durch Verbrennung } \\
\text { gefunden }\end{array}$} \\
\hline & \multicolumn{2}{|c|}{ Theoretisch } & \multicolumn{2}{|c|}{ nach Carius } & \multicolumn{2}{|c|}{$\begin{array}{l}\text { Gewichts- } \\
\text { analytisch }\end{array}$} & \multicolumn{2}{|c|}{$\begin{array}{c}\text { Mass- } \\
\text { analytisch }\end{array}$} \\
\hline & gr. & $\%$ & gr. & $\%$ & gr. & $\%$ & gr. & $\%$ \\
\hline $\begin{array}{l}\text { Jodoform } \\
\text { rein, } \mathrm{CHJ}_{3}\end{array}$ & & & & & & & & \\
\hline $0 \cdot 5556$ & $0 \cdot 5373$ & $96 \cdot 70$ & - & - & $0 \cdot 5375$ & $96 \cdot 74$ & $一$ & 一 \\
\hline 0.5542 & $0 \cdot 5359 \mid$ & $96 \cdot 70$ & - & - & 0.5361 & $96 \cdot 72$ & 一 & - \\
\hline $0 \cdot 3254$ & $0 \cdot 3147$ & $96 \cdot 70$ & - & 一 & - & - & $0 \cdot 3152$ & $96 \cdot 86$ \\
\hline $\begin{array}{l}\text { Jodoform } \\
\text { käuflich }\end{array}$ & & & & & & & & \\
\hline 0.3292 & - & - & $0 \cdot 3156$ & $95 \cdot 88$ & - & - & - & 一 \\
\hline 0.3954 & - & - & - & - & $0 \cdot 3795$ & $95 \cdot 98$ & 一 & - \\
\hline $0 \cdot 3268$ & - & - & - & - & $0 \cdot 3136$ & $95 \cdot 96$ & - & - \\
\hline $\begin{array}{l}\text { Jodmethyl } \\
\text { rein, } \mathrm{CH}_{3} \mathrm{~J} \text {. }\end{array}$ & & & & & & & & \\
\hline 0.5060 & $0 \cdot 4525$ & $89 \cdot 43$ & - & - & $0.4523 \mid$ & $89 \cdot 39$ & - & - \\
\hline $0 \cdot 4806$ & $0 \cdot 4298$ & $89 \cdot 43$ & 一 & - & - & - & $0 \cdot 4305$ & $89 \cdot 58$ \\
\hline $\begin{array}{l}\text { Jodäthyl } \\
\text { käuflich }\end{array}$ & & & & & & & & \\
\hline $0 \cdot 5034$ & - & - & $0 \cdot 4069$ & $80 \cdot 81$ & -1 & - & - & - \\
\hline $0 \cdot 2208$ & - & - & - & - & $0 \cdot 1783$ & $80 \cdot 75$ & - & - \\
\hline $0 \cdot 3168$ & - & 一 & - & - & $0 \cdot 2557$ & $80 \cdot 70$ & - & - \\
\hline $\begin{array}{c}\text { Jodumyl } \\
\text { rein, } \mathrm{C}_{5} \mathrm{H}_{1 \mathrm{I}} \mathrm{J}\end{array}$ & & & & & & & & \\
\hline $0 \cdot 3346$ & 0.2145 & $64 \cdot 11$ & - & - & $0 \cdot 2147$ & $64 \cdot 17$ & - & - \\
\hline $0 \cdot 3478$ & $0 \cdot 2230$ & $64 \cdot 11$ & - & - & - & - & $0 \cdot 2232$ & $64 \cdot 16$ \\
\hline $\begin{array}{l}\text { Trijodphenol } \\
\text { rein, } \mathrm{C}_{6} \mathrm{H}_{3} \mathrm{~J}_{3} \mathrm{O} \text {. }\end{array}$ & & & & & & & & \\
\hline $0 \cdot 4174$ & $0 \cdot 3368$ & $80 \cdot 70$ & $0 \cdot 3356$ & $80 \cdot 41$ & $1--$ & - & $=$ & - \\
\hline $0 \cdot 4750$ & $0 \cdot 3833$ & $80 \cdot 70$ & - & - & $0 \cdot 3825$ & $80 \cdot 53$ & - & $\cdots-$. \\
\hline $0 \cdot 3960$ & $0 \cdot 3196$ & $80 \cdot 70$ & - & - & - & - & $0 \cdot 3190$ & $80 \cdot 55$ \\
\hline
\end{tabular}

Specielles Verfahren bei Bromverbindungen.

Die Bromverbindungen verbrennen auch ziemlich leicht, desshalb benützt man ein Verbrennungsrohr von gleichen Dimensionen und gleicher Füllung wie beim Jod. Als Absorptionsfluissigkeit ist nur eine Mischung von Wasserstoffsuperoxyd und 
Ammoniak anwendbar, welche in derselben Menge wie beim Jod verwendet werden.

\begin{tabular}{|c|c|c|c|c|c|c|}
\hline \multirow{3}{*}{$\begin{array}{l}\text { Einwage } \\
\text { in } \\
\text { Grammen }\end{array}$} & \multicolumn{4}{|c|}{ Bromgehalt } & \multirow{2}{*}{\multicolumn{2}{|c|}{$\begin{array}{c}\text { Durch } \\
\text { Verbrennung } \\
\text { gefunden }\end{array}$}} \\
\hline & \multicolumn{2}{|c|}{ Theoretisch } & \multicolumn{2}{|c|}{ nach Carius } & & \\
\hline & gr. & $\%$ & gr. & $\%$ & gr. & $\%$ \\
\hline $\begin{array}{l}\text { Bromoform } \\
\text { rein, } \mathrm{CHBr}_{3} \text {. }\end{array}$ & & & & & & \\
\hline 0.4870 & $0 \cdot 4620$ & $94 \cdot 86$ & 0.4619 & $94 \cdot 85$ & - & $\ldots$ \\
\hline 0.4860 & $0 \cdot 4610$ & 94.86 & 0.4607 & $94 \cdot 80$ & - & - \\
\hline 0.4026 & $0 \cdot 3819$ & $94 \cdot 86$ & - & - & $0 \cdot 3807$ & $94 \cdot 57$ \\
\hline $0 \cdot 3062$ & $0 \cdot 2905$ & 94.86 & - & - & $0 \cdot 2893$ & $94 \cdot 50$ \\
\hline $\begin{array}{l}\text { Bromäthyl } \\
\text { käuflich }\end{array}$ & & & & & & \\
\hline $0 \cdot 3664$ & 一 & - & $0 \cdot 2676$ & $73 \cdot 04$ & - & - \\
\hline $0 \cdot 3410$ & - & - & - & - & $0 \cdot 2479$ & $72 \cdot 70$ \\
\hline $0 \cdot 4450$ & - & - & - & - & $0 \cdot 3226$ & $72 \cdot 50$ \\
\hline $\begin{array}{l}\text { Brombenzol } \\
\text { rein, } \mathrm{C}_{6} \mathrm{H}_{5} \mathrm{Br} \text {. }\end{array}$ & 0.1506 & $50 \cdot 94$ & 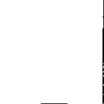 & 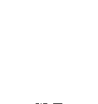 & & \\
\hline $\begin{array}{l}0.2956 \\
0.3394\end{array}$ & $0 \cdot 1729$ & $\begin{array}{l}50 \cdot 94 \\
50 \cdot 94\end{array}$ & - & - & $\begin{array}{l}0.1502 \\
0.1729\end{array}$ & $\begin{array}{l}50 \cdot 81 \\
50 \cdot 95\end{array}$ \\
\hline $\begin{array}{l}\text { Dibrombenzol } \\
\text { rein, } \mathrm{C}_{6} \mathrm{H}_{4} \mathrm{Br}_{2}\end{array}$ & & & & & & \\
\hline $0 \cdot 3688$ & $0 \cdot 2500$ & $67 \cdot 78$ & - & - & 0.2508 & $67 \cdot 86$ \\
\hline Tribromphenol & 0.2101 & - & - & - & $0 \cdot 2108$ & ber 85 \\
\hline $\begin{array}{r}\text { rein? } \mathrm{C}_{6} \mathrm{H}_{3} \mathrm{Br}_{3} \mathrm{O} \\
0.2748\end{array}$ & $0 \cdot 1992$ & $72 \cdot 50$ & 0.1988 & $72 \cdot 34$ & - & - \\
\hline $0 \cdot 3800$ & $0 \cdot 2755$ & $72 \cdot 50$ & - & - & 0.2738 & $72 \cdot 04$ \\
\hline $0 \cdot 3772$ & 0.2735 & $72 \cdot 50$ & - & - & $0 \cdot 2716$ & $72 \cdot 02$ \\
\hline $\begin{array}{l}\text { Bromwasserstoff- } \\
\text { saures Tetrabrom- } \\
\text { methylaurin, rein, } \\
\begin{array}{c}\left(\mathrm{C}_{20} \mathrm{H}_{12} \mathrm{Br}_{4} \mathrm{O}_{31} \mathrm{HBr}\right) \\
+\mathrm{aq}\end{array}\end{array}$ & & & & & & \\
\hline 0.2012 & $0 \cdot 1119$ & $55 \cdot 62$ & - & - & $0 \cdot 1118$ & $55 \cdot 58$ \\
\hline $0 \cdot 3042$ & $0 \cdot 1692$ & $55 \cdot 62$ & - & 一 & $0 \cdot 1694$ & $55 \cdot 69$ \\
\hline
\end{tabular}

Wenn die Substanz bromreich ist, so lässt sich das Brom an der Farbe der Verbrennungsproducte erkennen. 
Wenn das Wasserstoffsuperoxyd in unzureichender Menge angewendet wird, so bildet sich wahrscheinlich etwas Bromat und dieses wird durch späteren Zusatz von Hyperoxyd nicht redueirt; es ist also eine Reparatur wie bei Jod nicht möglich.

Die vereinigten Absorptions- und Waschflüssigkeiten werden wie beim Jod concentrirt und das Brom in gleicher Weise gefällt.

Aus den angeführten Beleganalysen ist zu ersehen, dass die Verbrennungsmethode auch lier gute Resultate gibt.

Specielles Verfahren bei Chlorverbindungen.

Die Verbrennung von Jod- und Bromverbindungen geht bei erlangter Übung sehr gut von Statten und beansprucht nach der Natur der Substanz $1 / 2$ bis 2 Stunden in den weitesten Grenzen. Weit schwieriger gestaltet sich die Sache bei Chlorverbindangen, welche im Allgemeinen schwerer verbrennen und ausserdem wird das Chlor oder die Salzsäure von dem Platin hartnäckig zurïckgehalten, so dass die Verdrängung dieser Gase durch den Sauerstoff viel Zeit beansprucht und doch nicht bis auf Null beendet werden kaun.

Kopfer konnte Paradichlorbenzol nur unter Mitanwendung von Silber verbrennen, um anf diese Art die Menge des Kohlenstoffes und des Wasserstoffes zu bestimmen. ${ }^{1}$ Will man eine totale Verbrennung ohne Silber zuwege bringen, so muss man eine grössere Menge von platinirtem Quarz, eine merklich höhere Temperatur und somit auch ein längeres und weiteres Verbrennungsrolre anwenden.

Die Dimensionen desselben in Centimetern sind aus folgender Zeichnung (Fig. 9) zu entnehmen.

Fig. 9.

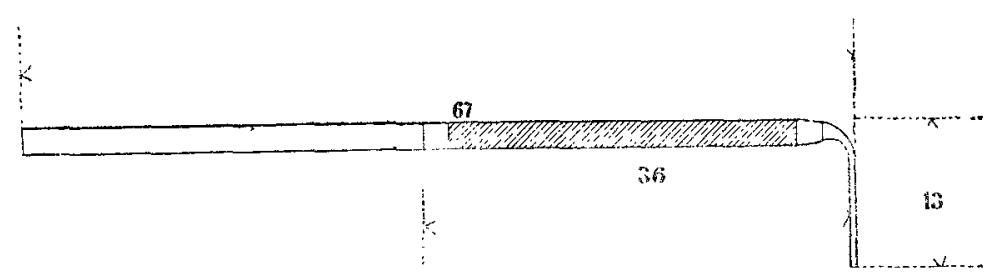

Die Weite des Glasrohres hat etwa $16 \mathrm{Mm}$. zu betragen; dies hat zur Folge, dass die flichtigen Producte verhältnissmässig

1 Zeitschrift für analyt. Chemie, 17. Jahrg. S. 23. 
längere Zeit einer höheren Temperatur ausgesetzt bleiben. Als Absorptionsflüssigkeit verwendet man Wasserstoffsuperoxyd und Ammoniak in Mengen wie bei Jod und Brom. Ist Ersteres nicht ausreichend, so bildet sich etwas Chlorat, welches durch nachträglichen Zusatz von Hyperoxyd nicht reducirt wird; daher auch hier eine Reparatur ausgeschlossen ist.

Ist die Verbrennung beendet, so muss man, wie schon früher bemerkt wurde, noch so lange Sauerstoff hindurchleiten, bis die Gasblasen, welche aus der Fliissigkeit emporsteigen, nur eine sehr schwache Nebelbildung verursachen. Dies dauert circa eine Stunde, somit erfordert die Verbrennung hier einen Zeitaufwand von 2 bis 3 Stunden. Salzsaure organische Verbindungen ergeben keine solchen Schwierigkeiten. Salzsaures Methylaurin z. B. verbrannte leicht und die Nebelbildung schwand gänzlich bei eirihalbstündigem Durchleiten von Sauerstoff nach beendeter Verbrennung.

In nachstehender Tabelle sind die mit Chlorverbindungen erzielten Resultate verzeichnet:

\begin{tabular}{|c|c|c|c|c|c|c|}
\hline \multirow{3}{*}{$\begin{array}{l}\text { Einwage } \\
\text { in } \\
\text { Grammen }\end{array}$} & \multicolumn{4}{|c|}{$\mathrm{Chlorg}$ elhalt } & \multirow{2}{*}{\multicolumn{2}{|c|}{$\begin{array}{c}\text { Durch } \\
\text { Verluronnung } \\
\text { gefunden }\end{array}$}} \\
\hline & \multicolumn{2}{|c|}{ 'Theoretiseh } & \multicolumn{2}{|c|}{ nach Carius } & & \\
\hline & gr. & $\%$ & gr. & $\%$ & gr. & $\%$ \\
\hline $\begin{array}{c}\text { Monochlorbenzol } \\
\text { käuflich }\end{array}$ & & & & & & \\
\hline $0 \cdot 2144$ & - & - & 0.0668 & $31 \cdot 15$ & - & - \\
\hline $0 \cdot 3362$ & - & - & - & 一 & $0 \cdot 0988$ & $30 \cdot 92$ \\
\hline $\begin{array}{l}\text { Dichlorbenzol } \\
\text { rein, } \mathrm{C}_{6} \mathrm{H}_{4} \mathrm{Cl}_{2} \text {. }\end{array}$ & & & & & & \\
\hline $0 \cdot 2954$ & $0 \cdot 1426$ & $48 \cdot 27$ & 0.1425 & $48 \cdot 24$ & - & - \\
\hline $\begin{array}{l}0.2934 \\
0.2758\end{array}$ & $\begin{array}{l}0 \cdot 1416 \\
0.1331\end{array}$ & $\begin{array}{l}48 \cdot 27 \\
48 \cdot 27\end{array}$ & - & - & $\begin{array}{l}0 \cdot 1409 \\
0 \cdot 1326\end{array}$ & $\begin{array}{l}48 \cdot 01 \\
48 \cdot 06\end{array}$ \\
\hline $\begin{array}{l}\text { Salzsäurehältiges } \\
\text { Methylaurin, rein, } 1 \\
\qquad \begin{array}{c}\left(\mathrm{C}_{23} \mathrm{H}_{24} \mathrm{ClO}_{4}\right) . \\
0.7928\end{array}\end{array}$ & 0.0721 & $9 \cdot 10$ & - & - & 0.0728 & $9 \cdot 18$ \\
\hline & & & & & & \\
\hline
\end{tabular}

1 Dieses Präparat wird erhalten, indem man eine weingeistige Lösung von Methylaurin mit Salzsäure versetzt und krystallisiren läisst. Die Formel ist durch Elementaranalyse festgestellt, die Constitution jedoch unbekanut. 
Einige Erfahrungen uber die Kalkmethode zur Bestimmung der Halogene.

Es haben gewiss schon viele Analytiker die unangenehme Erfahrung gemacht, dass es sehr schwer ist, einen chlor- and schwefelfreien Kalk zu beschaffen. Statt Kalk kann man jedoch Magnesia anstandslos verwenden.

Die sogenannte Magnesia usta enthält gewöhnlich gar kein Chlor oder höchstens unbestimmbare Spuren desselben. Sollte aber der Chlorgehalt bestimmbar sein, so kann man denselben durch Waschen mit kochendem Wasser entziehen. Diese Arbeit wird am besten in der Weise durchgeführt, dass man die Magnesia 1 bis 2 Mal mit Wasser auskocht, die Flüssigkeit abgiesst und den Riuckstand auf einem Filter ausbreitet und mit kochendem Wasser so lange wäscht, bis sich derselbe chlorfrei erweist.

Die (eventuell gereinigte) Magnesia muss längere Zeit in Platingefässen gegltiht werden, um das Wasser und die Kohlensäure zu entfernen. Da sie viel leichter ist als der Kalk, so braueht man für eine Verbrennung weniger. Die Kalk- oder Magnesiamethode hat die Unannehmlichkeit, dass die organische Substanz Kohle zurïcklässt, welche abfiltrirt und sehr gut gewaschen werden muss; ferner dass bei geringerer Hitze eine unvollständige Verkohlung, also eine Braunfärbung der Lösung eintritt. Es wäre sehr leicht möglich, diesem Übelstande dadurch zu begegnen, wenn man am Schlusse Sauerstoff durchleiten würde; nur müsste dem Rohre eine hiefür passende Gestalt gegeben werden. Die Anwendung des Sauerstoffes hätte ausserdem noch den Vortheil, dass etwa gebildetes Cyan zerstört wird. Bedenklich dagegen wäre die Verwendung desselben bei der Analyse jodhaltiger Substanzen, da die Möglichkeit einer Bildung von Jodat nicht ausgeschlossen erscheint.

Über die Anwendbarkeit der Verbrennungsmethode zur Bestimmung des Schwefels organischer Körper.

Es ist selbstverständlich, dass sich die früher geschilderte Methode mit allen ihren Apparaten eben so gut zur Schwefelbestimmung eignet. Als Absorptionsflüssigkeit hat man eine 
558 Zulkowsky u. Lepéz. Z. Best. d. Halogene org. Körper.

Mischung von Wasserstoffsuperoxyd und Ammoniak oder bromirte Kalilauge zu benttzen. Das Trommsdorff'sche Hyperoxyd enthält bestimmbare Spuren von Schwefelsäure, man muss also mit gemessenen Mengen arbeiten. Die zweite Absorptionsflüssigkeit bereitet man auf folgende Art:

Es werden $100 \mathrm{Grm}$. mit Alkohol gereinigtes, d. h. schwefelfreies Ätzkali in Wasser gelöst und 100 Grm. Brom unter Abkühlung eintropfen gelassen. Die wirksamen Bestandtheile sind unterbromigsaures Kali und überschüssiges Ätzkali. Ersteres oxydirt das Schwefeldioxyd und letzteres bindet die Schwefelsäure, das Schwefeltrioxyd und die Kohlensäure. Diese Flüssigkeit wird auf 1 Liter verdünnt und für je $0 \cdot 1 \mathrm{Grm}$. Schwefel $6 \mathrm{Cc}$. hievon an ge wendet. Diese Menge gewährt eine vierfache Sicherheit. Das auf tretende Schwefeltrioxyd ist merkwürdigerweise schwer verdichtbar und es muss derselbe Absorptionsapparat wie in Fig. 1 verwendet werden, um es völlig zurïckzuhalten. Die auftretende Schwefelsäure verdichtet sich in der abgebogenen Spitze in öligen Tropfen und muss von Zeit zu Zeit von den oberen Lagen weggeheizt werden. $\mathrm{Zu}$ diesem $\mathrm{Zwecke}$ bestreicht man diese Stellen mit der Flamme eines Brenners.

Wenn die öligen Tropfen nicht mehr auftreten und die Nebelbildung völlig verschwunden ist, so ist der Versuch beendet. 